\title{
Point-of-care ultrasound: a protean opportunity for perioperative care
}

\author{
Gregory L. Bryson, MD, FRCPC, MSc (1) • Hilary P. Grocott, MD, FRCPC, FASE
}

Received: 15 December 2017/ Accepted: 17 December 2017/Published online: 19 January 2018

(C) Canadian Anesthesiologists' Society 2018

In the Odyssey, Menelaus was becalmed on the island of Pharos while returning home from the Battle of Troy. Idothea, the daughter of Proteus, the old man of the sea, pitied Menelaus. Proteus was equally renowned for his abilities to both change his shape and foresee the future. Idothea told Menelaus, "If you can snare [Proteus] and hold him tight, he will tell you about your voyage, what courses you are to take, and how you are to sail the sea so as to reach your home". "So what does ancient Greek mythology have to do with ultrasound?

Like Proteus, non-invasive imaging at the point of care by using ultrasound is a constantly changing and powerful resource. Transthoracic echocardiographic (TTE) measurement of cardiac structure and function, obtained by specialist echocardiographers, has informed perioperative medicine for decades. Written reports, often taking days to appear in the chart, provided guidance for the perioperative journey. It wasn't until the late 1980s, when transesophageal echocardiography (TEE) entered into cardiac anesthesia practice, that anesthesiologists both obtained and interpreted ultrasound images in real time permitting dynamic, ongoing information during surgery and in the critical care unit. As more portable and higher fidelity equipment became

G. L. Bryson, MD, FRCPC, MSc ( $\varangle)$

Department of Anesthesiology and Pain Medicine, University of Ottawa, 1053 Carling Avenue, Box 249C, Ottawa, ON K1Y 4E9, Canada

e-mail: glbryson@toh.ca

G. L. Bryson, MD, FRCPC, MSc

Clinical Epidemiology Program, Ottawa Hospital Research Institute, Ottawa, ON, Canada

H. P. Grocott, MD, FRCPC, FASE

Department of Anesthesia and Perioperative Medicine,

University of Manitoba, Winnipeg, MB, Canada available, ultrasound found its way into regional anesthesia and vascular access applications. With the continued improvement in ultrasound technology, further expansion into increasingly diverse point-of-care applications and spread among multiple specialty areas of medicine rapidly followed. As a result, point-of-care ultrasound (POCUS) is now a protean term, changing its shape to suit the needs of its user. This special issue of the Journal highlights many of these diverse POCUS applications and the differing needs of their users.

Ultrasonography of the heart, great vessels, ${ }^{2}$ lungs, pleura, ${ }^{3}$ and abdomen in trauma ${ }^{4}$ are core curriculum elements common to many medical specialties. Critical care and emergency medicine have incorporated these skills as competencies within their basic Royal College of Physicians and Surgeons of Canada (RCPSC) residency training programs. ${ }^{5}$ Like Proteus, POCUS will shift its shape to respond to it users. There is an increasing demand for additional training for limited transesophageal imaging of thoracic contents among anesthesiologists ${ }^{6}$ and other specialists ${ }^{5}$ who might use this technology to guide resuscitation. Anesthesiologists are evolving POCUS applications relevant to their practices including ultrasonography of the airway ${ }^{7}$ and imaging of the stomach and its contents. ${ }^{8}$ Astute readers will note that our series featured here in this special issue's contents excludes USguided vascular access in adults (ubiquitous) and US-guided regional anesthesia (highly specialty specific), which most user communities do not see as POCUS, although the technology and user training share many similarities. This diversity of uses and user communities makes providing a simple one-size-fitsall training in POCUS a significant challenge.

Which brings us to Idothea's suggestion that Proteus will reveal "what courses to take". As the barriers to POCUS use in practice are lowered, the education of both trainees and practicing specialists becomes increasingly important. 
Standardization of basic and advanced skills, both procedural and cognitive, is a subject of considerable diversity within and across medical specialities, and highly inconsistent within anesthesiology. ${ }^{9}$ The RCPSC specialty committees will have to wrestle with what skills will be taught, by whom, and at what stage of training. The Journal has already highlighted the barriers to teaching cardiac ultrasound among Canadian anesthesiology training programs including human resources, expertise, and equipment. ${ }^{10}$ If we are to integrate POCUS within our competency-based RCPSC training programs we will have to answer a number of difficult questions. What are the milestones and entrustable professional activities associated with POCUS $?^{11}$ Do we have sufficient trained faculty to provide both didactic and bedside education? Are POCUS skills transferable between adult and pediatric populations? $^{12}$ If POCUS skills are fundamental entrustable professional activities for graduating specialists, how do we train thousands of providers already in practice? What is the role of technology in distributing training and maintenance of competence? ${ }^{13}$ What, if any, quality management will be put in place within hospitals to ensure the competence of the operator? Does the RCPSC engage in the evaluation and maintenance of competence in POCUS skills? And perhaps the greatest challenge, while POCUS assists providers in bedside decision-making, do those decisions actually improve patient outcome? ${ }^{14}$ These are tough questions and we'll need to wrestle with them to secure the place of POCUS in perioperative medicine.

The Journal hopes that this series of articles provides both an impetus to explore the uses of POCUS in our practice and a reliable source of information on the topic. This rapidly shifting and evolving knowledge base requires the engagement of user communities from the bedside all the way to the RCPSC. Point-of-care ultrasound offers an incredible opportunity for interdisciplinary collaboration that we should be eager to explore both in the classroom and at the bedside. Like Proteus, POCUS will show us the future if we are ready to grapple with it and grasp its changing nature.

\section{L'échographie au chevet: une occasion protéiforme pour les soins périopératoires}

Dans l'Odyssée, le roi Ménélas resta immobilisé sur l'île de Pharos alors qu'il retournait chez lui après la Guerre de Troie. Idothée, fille de Protée, le 'Vieillard de la mer', prit pitié de Ménélas. Protée était tout aussi connu pour son don de métamorphose que pour celui de prophétie. Idothée dit à Ménélas, "Si tu peux le saisir [Protée] dans quelques embûches, il t'enseignera la route, la longueur du voyage, le moyen de retourner dans ton foyer et comment tu pourras naviguer sur la mer poissonneuse $»{ }^{1}$ Quel est donc le rapport entre la mythologie de la Grèce antique et l'échographie?

Tout comme Protée, l'imagerie non invasive pratiquée au chevet à l'aide de l'échographie est une ressource puissante et en constante évolution. Depuis des dizaines d'années, les mesures des structures et de la fonction cardiaques par échocardiographie transthoracique (ETT), obtenues par des échocardiographistes spécialisés, guident la médecine périopératoire. Les rapports écrits, prenant souvent plusieurs jours avant d'apparaître au dossier du patient, servaient de guides dans le périple périopératoire. Ce n'est qu'à la fin des années 1980, lorsque l'échocardiographie transœsophagienne (ETO) a fait son apparition dans la pratique de l'anesthésie cardiaque, que les anesthésiologistes ont obtenu et interprété des images d'échographie en temps réel, ce qui a permis d'avoir des informations dynamiques et en continu pendant la chirurgie et à l'unité des soins intensifs. Au fur et à mesure que l'équipement est devenu plus portable et de plus haute résolution, l'échographie a fait son chemin dans l'anesthésie régionale et les applications d'accès vasculaire. Grâce aux améliorations constantes des technologies échographiques, cette modalité fait aujourd'hui l'objet d'une rapide expansion dans des applications au chevet de plus en plus variées et dans de nombreux domaines spécialisés de la médecine. Ainsi, l'échographie au chevet (PoCUS) est aujourd'hui un terme protéiforme, se métamorphosant pour répondre aux besoins de son utilisateur. Cette édition spéciale du Journal présente bon nombre des diverses applications de l'échographie au chevet et des besoins distincts de ses utilisateurs.

L'échographie du cœur, des grands vaisseaux, ${ }^{2}$ des poumons, de la plèvre ${ }^{3}$ et de l'abdomen en traumatologie ${ }^{4}$ sont des éléments centraux des programmes de formation et sont partagés par plusieurs spécialités médicales. La médecine des soins intensifs et la médecine d'urgence ont intégré ces compétences dans leurs programmes de formation de base des résidents du Collège royal des médecins et chirurgiens du Canada (CRMCC). ${ }^{5}$ Tout comme Protée, l'échographie au chevet se métamorphosera pour répondre à ses utilisateurs. La demande augmente pour une formation approfondie en imagerie transœsophagienne limitée du contenu thoracique pour les anesthésiologistes ${ }^{6}$ et d'autres spécialités, ${ }^{5}$ lesquelles pourraient se servir de cette technologie pour guider une réanimation. Les anesthésiologistes font évoluer les applications de l'échographie au chevet pertinentes à 
leurs pratiques, notamment l'échographie des voies aériennes $^{7}$ et l'imagerie de l'estomac et de son contenu. ${ }^{8}$ Le lecteur attentif remarquera que notre série, présentée dans ce numéro spécial, n'aborde pas les accès vasculaires échoguidés chez l'adulte (qui sont omniprésents) et l'anesthésie régionale échoguidée (qui est ultra-spécialisée), car la plupart des communautés d'utilisateurs ne les considèrent pas comme de l'échographie au chevet, malgré le fait que la technologie et la formation partagent de nombreuses similitudes. En raison de cette variété d'utilisations et de communautés d'utilisateurs, il est très difficile d'offrir une formation simple et unique en échographie au chevet.

Ce qui nous ramène à la suggestion d'Idothée, selon laquelle Protée révélera « la route à suivre ». Alors que les obstacles à l'utilisation de l'échographie au chevet diminuent, la formation tant des personnes en formation que des spécialistes en pratique devient de plus en plus importante. La standardisation des compétences de base et avancées, tant au niveau procédural que cognitif, est remarquablement diversifiée, tant au sein de chaque spécialité médicale que d'une spécialité à une autre, et c'est le cas à l'intérieur même de l'anesthésiologie. ${ }^{9}$ Les comités de spécialité du CRMCC auront du travail avant de pouvoir déterminer quelles compétences enseigner, par qui, et à quelle étape de la formation. Le Journal a déjà présenté, par le passé, les obstacles à l'enseignement de l'échographique cardiaque dans les programmes de formation en anesthésiologie au Canada, notamment les problèmes de ressources humaines, d'expertise et de matériel. ${ }^{10} \mathrm{Si}$ nous souhaitons intégrer l'échographie au chevet au sein de nos programmes de formation fondés sur la compétence du CRMCC, nous devrons répondre à plusieurs questions difficiles. Quels sont les jalons et les actes professionnels non supervisés associés à l'échographie au chevet? ${ }^{11}$ Disposons-nous de suffisamment de personnes formées pour offrir à la fois une formation didactique et une formation au chevet? Les compétences en échographie au chevet sont-elles transférables des populations adultes aux populations pédiatriques? ${ }^{12} \mathrm{Si}$ les compétences en échographie au chevet sont des actes professionnels non supervisés fondamentaux pour les spécialistes terminant leur formation, comment formons-nous les milliers de professionnels pratiquant déjà l'anesthésie? Quel rôle joue la technologie dans la diffusion de la formation et le maintien des compétences? ${ }^{13}$ Le cas échéant, quel type de gestion de la qualité sera mis en place dans les hôpitaux afin de s'assurer des compétences des opérateurs? Faut-il impliquer le CRMCC dans l'évaluation et le maintien des compétences en échographie au chevet? Et, ce qui constituera peut-être le défi le plus important, alors que l'échographie au chevet aide les fournisseurs à prendre des décisions au chevet, dans quelle mesure ces décisions améliorent-elles véritablement le pronostic des patients? ${ }^{14}$ Voilà des questions difficiles auxquelles nous devrons répondre si nous désirons établir une fois pour toutes la position de l'échographie au chevet en médecine périopératoire.

Le Journal espère que cette série d'articles servira à la fois de tremplin pour explorer les utilisations de l'échographie au chevet et de source d'informations fiables sur le sujet. Cette base de connaissances en constante évolution nécessite l'implication de toutes les communautés d'utilisateurs, du chevet jusqu'au CRMCC. L'échographie au chevet constitue une occasion exceptionnelle de collaboration interdisciplinaire que nous devrions nous empresser d'explorer, tant dans les salles de classe qu'au chevet. Tout comme Protée, l'échographie au chevet nous montrera l'avenir - si nous sommes prêts à l'affronter et à saisir sa nature changeante.

Declaration of interests Drs Bryson and Grocott are the Deputy Editor-in-Chief and Editor-in-Chief, respectively, of the Canadian Journal of Anesthesia.

Editorial responsibility This submission was handled by Dr. Philip M. Jones, Associate Editor, Canadian Journal of Anesthesia.

Déclarations d'intérêt Les Drs Bryson et Grocott sont le rédacteur en chef adjoint et le rédacteur en chef, respectivement, du Journal canadien d'anesthésie.

Responsabilitè èditoriale Cet article a été traité par Dr Philip M. Jones, rédacteur adjoint, Journal canadien d'anesthésie.

\section{References}

1. Homer. The Odyssey. Book IV. Translated by Samuel Butler. The Internet Classics Archive by Daniel C. Stephenson. Web Atomics (C) 1994-2009. Available from URL: http://classics.mit. edu/Homer/odyssey.4.iv.html. Accessed Dec 2017.

2. Denault AY, Langevin S, Lessard MR, Courval JF, Desjardins G. Transthoracic echocardiographic evaluation of the heart and great vessels. Can J Anesth 2018. DOI: https://doi.org/10.1007/s12630018-1068-4.

3. Goffi A, Kruisselbrink R, Volpicelli G. The sound of air: point-ofcare lung ultrasound in perioperative medicine. Can J Anesth 2018. DOI: https://doi.org/10.1007/s12630-018-1062-x.

4. Pace J, Arntfield R. Focused assessment with sonography in trauma: a narrative review of concepts and considerations for anesthesiology. Can J Anesth 2018. DOI: https://doi.org/10.1007/ s12630-017-1030-x.

5. Millington SJ, Goffi A, Arntfield RT. Critical care echocardiography: a certification pathway for advanced users. Can J Anesth 2018. DOI: https://doi.org/10.1007/s12630-0181061-y. 
6. Fayad A, Shillcutt SK. Perioperative transesophageal echocardiography for non-cardiac surgery. Can J Anesth 2018. DOI: https://doi.org/10.1007/s12630-017-1017-7.

7. You-Ten KE, Siddiqui N, Teoh WH, Kristensen MS. Point-of-care ultrasound (POCUS) of the upper airway. Can J Anesth 2018. DOI: https://doi.org/10.1007/s12630-018-1064-8.

8. Perlas A, Arzola C, Van de Putte P. Point-of-care gastric ultrasound and aspiration risk assessment: a narrative review. Can J Anesth 2018. DOI: https://doi.org/10.1007/s12630-0171031-9.

9. Meineri M, Bryson GL, Arellano R, Skubas N. Core point-of-care ultrasound curriculum: what does every anesthesiologist need to know? Can J Anesth 2018. DOI: https://doi.org/10.1007/s12630018-1063-9.

10. Mizubuti $G$, Allard $R$, Ho AM, Cummings $M$, Tanzola $R C$. A survey of focused cardiac ultrasonography training in
Canadian anesthesiology residency programs. Can J Anesth 2017; 64: 441-2.

11. McCormick TJ, Miller EC, Chen R, Naik V. Acquiring and maintaining point-of-care ultrasound (POCUS) competence for anesthesiologists. Can J Anesth 2018. DOI: https://doi.org/10. 1007/s12630-018-1049-7.

12. Su E, Dalesio N, Pustavoitau A. Point-of-care ultrasound in pediatric anesthesiology and critical care medicine. Can J Anesth 2018. DOI: https://doi.org/10.1007/s12630-018-1066-6.

13. Choo EK, Chen R, Millington SJ, et al. Remote solutions for telementoring point-of-care ultrasound echocardiography: The RESOLUTE study. Can J Anesth 2017; 64: 1077-8.

14. Bainbridge D, McConnell B, Royse C. A review of diagnostic accuracy and clinical impact from the focused use of perioperative ultrasound. Can J Anesth 2018. DOI: https://doi. org/10.1007/s12630-018-1067-5. 